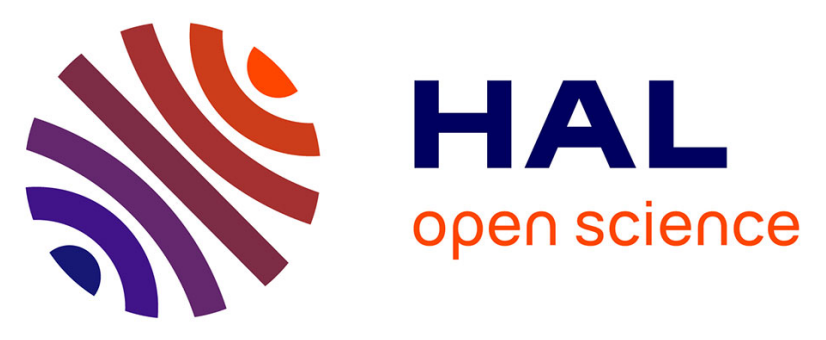

\title{
Real-Time Measurements of Ultrafast Spontaneous Modulation Instability in Optical Fiber
}

Benjamin Wetzel, Mikko Narhi, Cyril Billet, Jean-Marc Merolla, Shanti

Toenger, Thibaut Sylvestre, R Morandotti, Goëry Genty, Frédéric Dias, John Michaël Dudley

\section{To cite this version:}

Benjamin Wetzel, Mikko Narhi, Cyril Billet, Jean-Marc Merolla, Shanti Toenger, et al.. Real-Time Measurements of Ultrafast Spontaneous Modulation Instability in Optical Fiber. Nonlinear Optics 2019, Jul 2019, Waikoloa Beachn, Hawaii, United States. hal-03369004

\section{HAL Id: hal-03369004 https://hal.science/hal-03369004}

Submitted on 7 Oct 2021

HAL is a multi-disciplinary open access archive for the deposit and dissemination of scientific research documents, whether they are published or not. The documents may come from teaching and research institutions in France or abroad, or from public or private research centers.
L'archive ouverte pluridisciplinaire HAL, est destinée au dépôt et à la diffusion de documents scientifiques de niveau recherche, publiés ou non, émanant des établissements d'enseignement et de recherche français ou étrangers, des laboratoires publics ou privés. 


\title{
Real-Time Measurements of Ultrafast Spontaneous Modulation Instability in Optical Fiber
}

\author{
Benjamin Wetzel, ${ }^{1,2}$ Mikko Närhi, ${ }^{3}$ Cyril Billet, ${ }^{4}$ Jean-Marc Merolla, ${ }^{4}$ Shanti Toenger, ${ }^{4}$ \\ Thibaut Sylvestre, ${ }^{4}$ Roberto Morandotti, ${ }^{1}$ Goëry Genty, ${ }^{3}$ Frederic Dias ${ }^{5}$, John M. Dudley ${ }^{4}$ \\ ${ }^{1}$ Institut National de la Recherche Scientifique, Université du Québec, Varennes, Québec J3X 1S2, Canada \\ ${ }^{2}$ School of Mathematical and Physical Sciences, University of Sussex, Sussex House, Falmer, Brighton BN1 9RH, UK \\ ${ }^{3}$ Tampere University of Technology, Optics Laboratory, FI-33101 Tampere, Finland \\ ${ }^{4}$ Université de Franche-Comté, Institut FEMTO-ST, 25030 Besançon, France \\ ${ }^{5}$ School of Mathematical Sciences, University College Dublin, Belfield, Dublin 4, Ireland \\ Authore-mailaddress: b.wetzel@sussex.ac.uk; john.dudley@univ-fcomte.fr
}

\begin{abstract}
We report on the real-time observation of highly-localized structures and rogue waves spontaneously emerging from noise during fiber propagation. Experimental results, obtained via a time-lens magnifier, are in good agreement with theory and numerical simulations.

OCIS codes: (190.0190) Nonlinear optics; (060.2310) Fiber optics; (060.7140) Ultrafast processes in fibers; (250.5530)

Pulse propagation and temporal solitons; (320.7100) Ultrafast measurements
\end{abstract}

\section{Introduction}

Modulation instability (MI) constitutes one of the most fundamental processes of nonlinear science, and develops in fiber optics when a weak perturbation on a continuous wave field experiences gain and evolves into highly-localized "breather" structures. Although MI has been widely studied over the last decades, there is still an intense interest in understanding its dynamics because, when triggered from noise, it generates high amplitude and statistically-rare "rogue waves" of importance in hydrodynamics and optics [1]. Due to experimental limitations, however, directly observing the ultrafast instability dynamics of noise-driven spontaneous MI in optics is extremely challenging. Although real time observations of this fundamental and spontaneous process have been reported via spectral measurements [2,3], experimentally accessing the temporal properties of the instability is usually limited by the bandwidth capability of current electronic detection systems. In this paper, we use an ultrafast time-lens magnifier system to perform direct measurements of real time temporal structures in MI. Our results show an extended series of transient high intensity breather pulses emerging from noise, and our statistical analysis allows the presence of long tails (rare events) to be readily observed and compared with numerical and analytic predictions.

\section{Results}

Fig. 1(a) presents the typical optical field evolution obtained from numerical simulations of fiber propagation and showing the evolution of noise-driven MI in the temporal domain. The simulations use our experimental parameters where a narrowband $\mathrm{CW}$ laser at $1552 \mathrm{~nm}$ was injected in up to $20 \mathrm{~km}$ of single mode fiber $\left(\beta_{2}=-21.4\right.$ $\left.\mathrm{ps}^{2} \mathrm{~km}^{-1}, \beta_{3}=0.012 \mathrm{ps}^{3} \mathrm{~km}^{-1}, \gamma=1.2 \mathrm{~W}^{-1} \mathrm{~km}^{-1}\right)$.
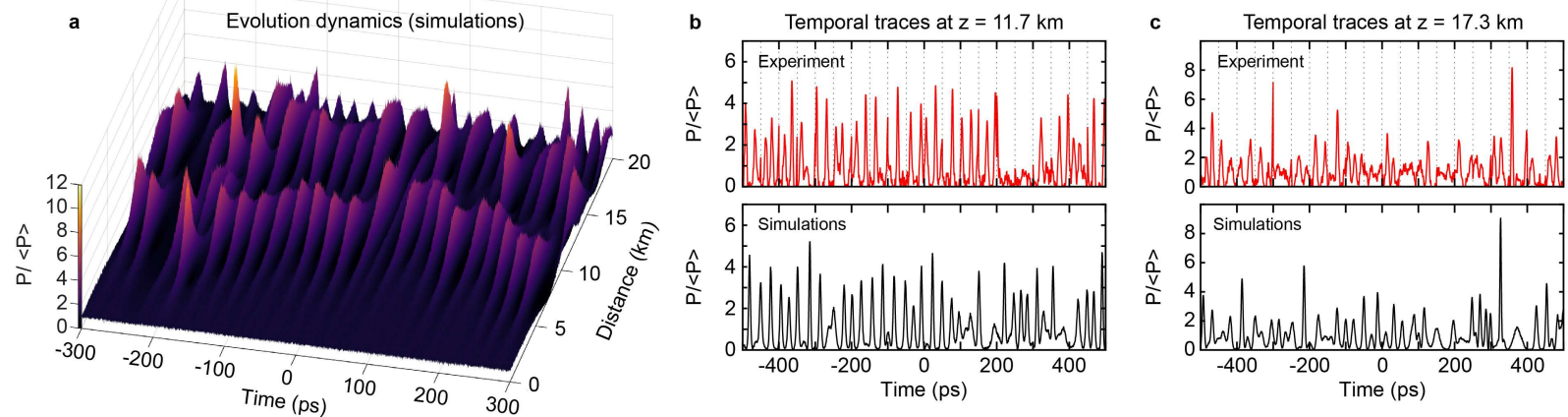

Fig. 1. (a) Single-shot simulation showing temporal evolution of spontaneous MI. (b,c) Results obtained at (a) $11.7 \mathrm{~km}$ and (b) $17.3 \mathrm{~km}$, showing intensity profiles from simulations (bottom, black) and experiments (top, red) as indicated. Plotted intensities P are normalized with respect to the average output background power $<\mathrm{P}>$ and plotted against time after rescaling to account for the effect of magnification. The experimental traces in $(b, c)$ are reconstructed form sequential measurement windows (see dashed lines between measurements) for visual clarity.

Here, we see the development from noise of a distinct series of temporal breather pulses that develop into a quasi-regular breather train at an initial compression point $(\mathrm{z} \sim 11 \mathrm{~km})$ before evolving chaotically with subsequent 
propagation. With input power $\mathrm{P}_{0}=0.7 \mathrm{~W}$, peak MI gain occurs at frequency $\Omega / 2 \pi \sim 43 \mathrm{GHz}$ where $\Omega^{2}=2 \gamma \mathrm{P}_{0} /\left|\beta_{2}\right|$ yielding (unstable) breather structures of $\sim 2$ ps duration. To observe these fluctuations experimentally, we use a time-lens magnification technique similar to that used to reveal rogue wave fluctuations in optical fiber turbulence [4]. In our setup, 2 ps MI breathers are stretched to $0.15 \mathrm{~ns}$ and readily captured in real-time using a $38 \mathrm{GHz}$ photoreceiver and ultrafast oscilloscope (see Ref. [5] for further details). Temporal intensity profiles extracted at two propagation distances typical of these qualitatively different breather localization dynamics are respectively presented in Fig. 1(b) and (c), comparing experimental results obtained from our time-lens characterization technique (top panel) with numerical simulations (bottom panel). One can readily observe the excellent qualitative agreement between the transient pulse trains recorded from sequential time-lens measurements and numerical predictions.

\section{Analysis}

In order to provide a more quantitative comparison with numerical and analytical predictions, we analyzed the experimentally recorded temporal traces by extracting individual pulse profiles and associated peak intensities and pulse durations. In Fig. 2, we present the intensity histogram of the chaotic MI pulse train at $17 \mathrm{~km}$ and typical temporal structures profiles retrieved from experimental measurements (red lines).
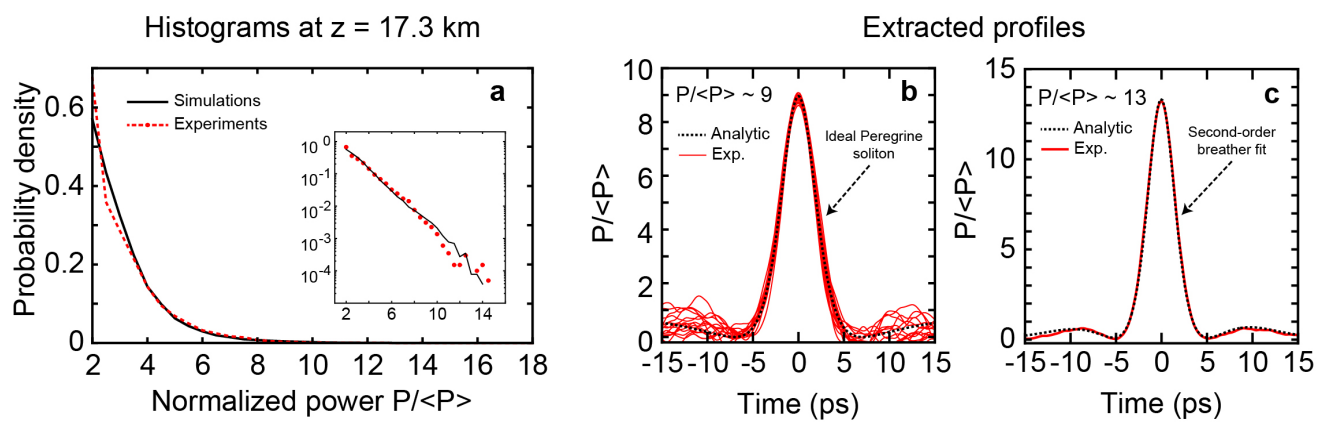

Fig. 2. (a) Histogram of the peak intensities (shown as normalized probability density) from the chaotic pulse trains at

$17.3 \mathrm{~km}$, comparing experiment (red) and simulation (black). The inset plots the histogram on semi-logarithmic axes. (b) Results extracting 10 intensity profiles at with $\mathrm{P} /\langle\mathrm{P}>\sim 9$ from experiment (red lines) are compared with the analytic Peregrine soliton (black dashed line). (c) A peak of higher intensity $\mathrm{P} /<\mathrm{P}>\sim 13$, extracted from experiments (red lines), is compared with a higher-order breather fit (black dashed line).

The results clearly show long tails in the peak intensity histogram (see Fig. 1(a)), associated with extreme event formation and in good agreement with analysis from stochastic numerical simulations (black). Fig. 2(b) shows a subset of the detected peaks with normalized intensity $\mathrm{P} /<\mathrm{P}>\sim 9$ which are superimposed and compared with the theoretical Peregrine soliton (PS) structure (dashed black line) [6], showing remarkable agreement with breather theory over the main intensity lobe region. An example of a temporal structure with higher peak intensity extracted from our experiment (red) is also illustrated in Fig. 2(c). The profile fit, obtained assuming a second-order breather solution and using the Darboux transformation (black line) [7], also provide excellent agreement with the pulse retrieved using our time-lens technique, thus illustrating the ability of the breather theory to describe the emergence of localized structures beyond their fundamental solutions. In addition, we observed that the relationship between the duration and power of spontaneously emerging temporal structures retrieved from experiment agrees well with simulations. They both cluster strongly around the theoretical prediction based on the analytic properties of NLSE elementary breathers and their collisions.

\section{Conclusions}

In conclusion, our results show how the optical time-lens can be used to provide direct insights into the properties of ultrafast modulation instability and to enable the direct capture of transient breather pulses emerging from noise. Besides this particular implementation, the technique has tremendous potential to shed light into the study of nonlinear instabilities and/or stochastic phenomena in a broader physical context.

\section{References}

[1] J. M. Dudley, F. Dias, M. Erkintalo, G. Genty, Nature Photon. 8, 755-764 (2014)

[2] B. Wetzel et al. Sci. Rep. 2, 882 (2012)

[3] D. R. Solli G. Herink, B. Jalali, C. Ropers, Nature Photon. 6, 463-468 (2012)

[4] P. Suret, R. El Koussai, A. Tikan, C. Evain, S. Randoux, C. Szwaj, and S. Bielawski, Nat. Commun. 7, 13136 (2016)

[5] M. Narhi et al. Nature Commun. 7, 13675 (2016)

[6] B. Kibler et al. Nature Physics 6, 790-795 (2010)

[7] S. Toenger et al. Sci. Rep. 5, 10380 (2015) 